\title{
Extracting the dielectric relaxation of water in thin Nafion membranes by terahertz spectroscopy
}

\author{
Xiaoran $\mathrm{Li}^{1}$, Decio.F. Alves-Lima ${ }^{1}$, Riccardo Degl'Innocenti ${ }^{1}$, Richard Dawson ${ }^{1}$, and Hungyen Lin ${ }^{1}$ \\ ${ }^{1}$ Department of Engineering, Lancaster University, LA1 4YW, United Kingdom
}

\begin{abstract}
Terahertz time domain spectroscopy (THz-TDS) has been demonstrated to quantify water uptake and retention properties of Nafion proton exchange membranes (PEMs). With a growing interest in thinner membranes, we present the analysis technique to cater for such samples and apply it to reveal it's water uptake and retention properties.
\end{abstract}

\section{INTRODUCTION}

Proton exchange membrane fuel cell has been widely applied on the energy source for electric vehicles [1] where the polymer electrolyte membrane is used for proton conduction, while simultaneously preventing electron transport and fuel cross-over. THz-TDS has previously been demonstrated to study water retention in a hydrated Nafion membrane [2] revealing the nature of water present, such as bulk, bound and free water. To date, most of the studies have been performed on relatively thick membranes eg $160 \mu \mathrm{m}$. At these thicknesss, existing algorithms [3], some of which are implemented in commercial parameter extraction software, can extract samples' optical constants. However difficulties arise for thin membranes eg. at $25 \mathrm{um}$ where the iterative algorithm fails to converge. Here we exploit the fact that dielectric response of these hydrated membranes follows a double debye model and develop a parametric based algorithm to extract the dielectric relaxation. We validate the analysis against [4] for the same membrane thickness and apply the algorithm to extract the water retention properties of thinner membrane at $25 \mathrm{um}$.

\section{METHODOLOGY}

THz-TDS measurements were performed using a TERA K15 (Menlo Systems, Germany). The hydrated Nafion 117 $(183 \mu \mathrm{m})$ and $211(25 \mu \mathrm{m})$ (Fuel Cell Store, TX, USA) were measured during a 25 minutes' dehydration process at ambient conditions $\left(\mathrm{T}=26^{\circ} \mathrm{C}, \mathrm{RH}=41 \%\right)$. $\mathrm{THz}$ waveforms were acquired at every minute interval from 5 averages. The parameteric algorithm assumes a double debye model, which is valid for these membranes [5]. To globally minimise the error function between measurement and the analytical model accounting for all etalons, a particle swarm optimisation is used. The double debye model is given by

$$
\varepsilon(\omega)=\varepsilon_{\infty}+\frac{\Delta \varepsilon_{1}}{\left[1+\left(i \omega \tau_{1}\right)\right]}+\frac{\Delta \varepsilon_{2}}{\left[1+\left(i \omega \tau_{2}\right)\right]}
$$

where $\varepsilon_{\infty}$ is infinite dielectric constant, $\Delta \varepsilon_{1}$ and $\Delta \varepsilon_{2}$ are defined as: $\Delta \varepsilon_{1}=\varepsilon_{S}-\varepsilon_{2}$ and $\Delta \varepsilon_{2}=\varepsilon_{2}-\varepsilon_{\infty}$. $\epsilon_{\mathrm{S}}$ is the static dielectric constant, $\epsilon_{2}$ is the intermediate value of the real part of the dielectric constant. $\tau_{1}$ is the slow relaxation time, which is regarded as $7.2 \mathrm{ps}[5]$ and $\tau_{2}$ is fast relaxation time.

\section{RESULTS}

Fig 1 shows the variation of the Debye parameters over time for both membranes. The trend of Debye parameters for two membranes is in general agreement with what is previously reported [5]. As expected, owing to a thinner membrane,
Nafion 211 dries out faster than Nafion 117 thus resulting in a faster stabalisation in the double Debye parameters.
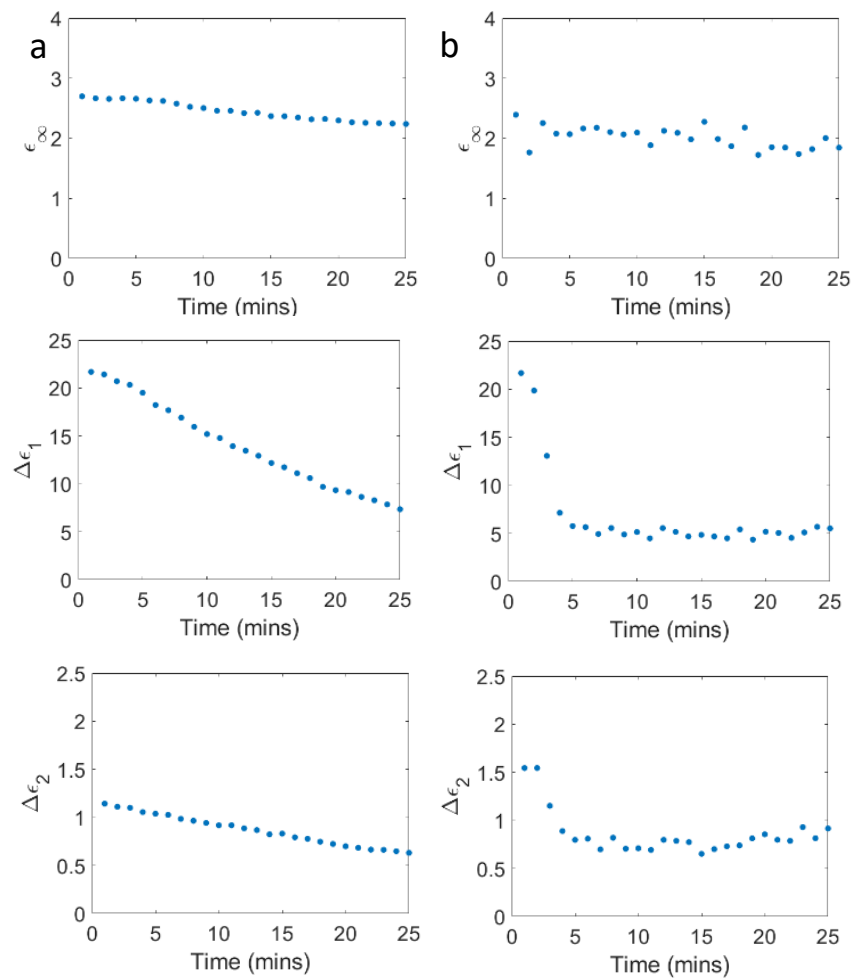

Fig. 1. The change of double Debye parameters of (a) Nafion 211 and (b) 117.

\section{ACKNOWLEDGEMENTS}

The authors acknowledge the financial support from UK EPSRC Grant EP/R019460/1 and H2FC Supergen Flexible Grant. The authors would also like to thank Johnson Mattheys helpful discussions.

\section{REFERENCES}

[1] J.-H. Wee, "Applications of proton exchange membrane fuel cell systems," Renewable and sustainable energy reviews, vol. 11, pp. 1720-1738, 2007.

[2] S. O. Yurchenko and K. I. Zaytsev, "Spectroscopy of Nafion in terahertz frequency range," Journal of Applied Physics, vol. 116, p. 113508, 2014.

[3] T. D. Dorney et al., "Material parameter estimation with terahertz timedomain spectroscopy," JOSA A, vol. 18, pp. 1562-1571, 2001.

[4] N. Devi et al., "Non-invasive macroscopic and molecular quantification of water in Nafion ${ }^{\circledR}$ and SPEEK Proton Exchange Membranes using terahertz spectroscopy," Journal of Membrane Science, vol. 588, p. 117183, 2019.

[5] N. Devi et al., "Tracking the hydration dynamics of Nafion fuel cell membranes using terahertz spectroscopy," in Terahertz, RF, Millimeter, and Submillimeter-Wave Technology and Applications XII, 2019, p. 109171R. 